\title{
A Presentation of the ERAS Project: The Danish Unit for Registration and Storage of Medical Research Data
}

The new Unit was established on november the $1^{\text {st }} 1996$. Years of work and planning by the Danish Data Archives finally led to its formation half a year ago. Sadly the initiator, the director of the Danish Data Archives, Per Nielsen, died less than two months after it's formation.

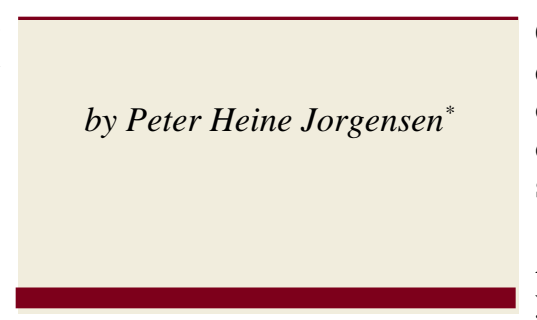

Consequently medical research studies constitutes only a small fraction, about $6 \%$ of the total contents, primarily in the form of social medicine and occupational health studies.

About 8000 articles are published every year by Danish medical researchers. Although not all of these articles represent a seperate research study, even a conservative estimate of 2 articles per study gives a yearly volume of 4000 studies. Add to this a considerable backlog of old studies and you begin to realize the magnitude of the task. This necessitates the development of new, timesaving procedures for registration and storage of data and documentation. An important consideration in the development work is to ensure that the high quality of registra tion and storage of data and documentation already attained at the Danish Data Archives is preserved.

The staff of the Unit at present consists of 3 persons:

The project leader, medical doctor and Ph.D. Kirsten Kyvik, who also is a member of the management group for the Danish Twin Register, senior researcher, medical doctor and specialist in community medicine and occupational health, Peter Heine Jorgensen and informatics assistant, Birgit Wich.

It is the first time that medical staff has been employed at the Danish Data Archives and indeed at the Danish State Archives. The reason of course being inherent in the nature of the project. The logic being that a medical staff is best suited to deal with medical research data and documentation. Furthermore it is expected that this will counteract any reluctancy or mistrust on the part of the donor of the medical research data. The employment of medical doctors in the staff also solves the confidentiality issue often associated with handling medical research data.

Another task presents itself to the Unit regarding confidentiality. The data may contain sensitive, identifiable information about the persons in the study base.

The Danish Data Protection Agency under the Danish Justice Department controls and regulates the legal aspects of data storage. The Agency has granted the Unit permisson 
to store sensitive data from medical research. Furthermore permission to release the original data and documentation to the donors is given. Thus the primary researchers are able to continue their study on the same study base, even years after completion of the first study. Naturally other researchers will only have access to the data in an anonymous form. The Unit is at present engaged in an effort to persuade the Data Protection Agency to accept that storage at the Unit can be regarded as being equal to deletion. It is hoped that this can be stipulated in the standard agreement made between the Agency and the medical researchers.

The target of the Unit is medical research in Denmark and existing as well as coming Danish medical researchers. A broad acceptance and support from the medical research community is instrumental in achieving a succesful result, ie. the creation of professional archives for medical research data. Consequently the initial action will focus on information and dialog. Agreement regarding submission of research data to the new unit will be investigated in accordance with the Danish medical research community,

The scope of the Danish Unit for Registration and Storage of Medical Research Data is to assist researchers and research institutions in making data and documentation from studies readily available. The new Unit will perform it's own research and development with the purpose of making the new archive as well functioning as possible, thus facilitating secondary analysis. The possibility of new crosslinks between the existing social science and historic data and the new medical research data represents a unique opportunity to perform secondary analysis bridging several major research fields.

The Units field of activitivity is at present confined to Danish medical research, but the findings could have an international bearing as the principles and procedures of the fully established Unit could be implemented in other countries. A more concerted implementation could be possible in the framework of the European Union.

* Paper presented at IASSIST/IFDO '1997 Conference, May 6th May 9th Odense, Denmark.

Peter Heine Jorgensen, M.D., Specialist in Community Medicine and Occupational Health. 